\title{
Reliable measurement of elastic modulus of cells by nanoindentation in an atomic force microscope
}

\author{
Z.L. Zhou ${ }^{1}$, A.H.W. Ngan ${ }^{1, \uparrow}$, B. Tang ${ }^{1,2}$, A.X. Wang ${ }^{3}$ \\ ${ }^{1}$ Department of Mechanical Engineering, the University of Hong Kong, Pokfulam \\ Road, Hong Kong, P.R. China \\ ${ }^{2}$ Department of Mechanical Engineering, King Abdullah University of Science and \\ Technology, Thuwal, Saudi Arabia \\ ${ }^{3}$ Department of Oral and Maxillofacial Surgery, the First Affiliated Hospital, Sun \\ Yat-Sen University, Guangzhou, China \\ "Corresponding author (email: hwngan@hku.hk)
}

\begin{abstract}
The elastic modulus of an oral cancer cell line UM1 is investigated by nanoindentation in an atomic force microscope with a flat-ended tip. The commonly used Hertzian method gives apparent elastic modulus which increases with the loading rate, indicating strong effects of viscoelasticity. On the contrary, a rate-jump method developed for viscoelastic materials gives elastic modulus values which are independent of the rate-jump magnitude. The results show that the rate-jump method can be used as a standard protocol for measuring elastic stiffness of living cells, since the measured values are intrinsic properties of the cells.
\end{abstract}

\section{Keywords}

Oral cancer cells; atomic force microscopy, nanoindentation, elastic modulus, viscoelasticity 


\section{Introduction}

Evidence suggests that metastasis may be related to the mechanical stiffness of the cancer cells [1-9]. For this reason, a lot of efforts have been made to investigate the mechanical properties of cells by methods including nanoindentation [5, 9-18], micro-pipette aspiration [1, 19-22] and micro-fluidic techniques [3, 23-26]. In general, the deformability of cancerous cell types was found to be different from that of their normal counterparts. Ward et al [1], Guck et al [3], Lekka et al [9] and Park et al [12] found that different sources of cancerous cells are more deformable than their normal counterparts, and Li et al [16] found that malignant breast cancer cells are softer than benign ones. On the other hand, Rosenbluth et al [13] and Zhang et al [20] concluded that the leukemia cell line HL60 [13] and hepatocellular carcinoma cells [20] are stiffer than normal. Whether cancerous cells are stiffer than normal may depend on the cell type and pathogeny of the disease, and the above observations by different researchers suggest that mechanical properties can be used to indicate the difference between cancerous cells and normal cells.

Among the methods commonly used for measuring mechanical behavior of cells, nanoindentation is by far the most popular since quantitative measures of the mechanical properties can be obtained directly without the need of time consuming post-experiment analysis such as finite-element modeling. Carrying out nanoindentation in an atomic force microscope (AFM), rather than a commercial nanoindenter, also has the advantage of enabling scanning imagining on the same platform as the mechanical indentation, as well as providing very accurate spatial control of the indent location with submicron precision. Environmental fluid chambers for AFM are also well developed and available at low costs, thus enabling conditions close to in vivo to be achievable. In a typical AFM nanoindentation experiment [5, 9-18], the load $(P)$ vs displacement $(\delta)$ data are gathered while the cell is indented at a fixed displacement rate. The $P-\delta$ data are often fitted by the Hertzian model of elastic contact [27] to generate an elastic modulus. Since the Hertzian model assumes the sample to be purely linear elastic, while a cell consists of a cytoplasm-membrane-cytoskeleton structure which does not behave in a purely linear elastic manner, the Hertzian model is almost definitely a wrong description of the nanoindenation response. In fact, Li et al. [16] found that the elastic modulus of breast cancer cells measured by such a method increased with the loading rate, resembling the behavior of viscoelastic materials [28]. The fact that the modulus so obtained is not an intrinsic property of the specimen, but is dependent on the way the experiment is carried out, precludes reliable comparison between different types of cells or the same type of cells treated with different protocols, or between results gathered from different laboratories. 
The cytoplasm-membrane-cytoskeleton nature of living cells should be more accurately described by viscoelasticity, than by purely linear elasticity as in the Hertzian model. Accurate measurement of the elastic modulus of a viscoelastic material by nanoindentation needs to have viscous effects properly considered [29, 30]. In this paper, we investigate the validity of a recently developed rate-jump protocol for carrying out AFM nanoindentation [31] on a cell line. Instead of running the test at a constant displacement rate as in the Hertzian method, in this protocol a step change in the displacement rate is imposed, which results in a step change in the load rate. Theoretical considerations [32] have shown that the relationship between these two step changes yields an elastic modulus which is intrinsic of any power-law viscoelastic material.

In addition to considering viscoelastic rather than elastic responses in the measurement protocol, any attempt to use a measured elastic modulus as a signature of a cell's state should also take into account consideration of the phase in the cell cycle, cell mobility and cell migration, which are factors that can affect the cytoskeleton [33-39]. Thus, indenting a cell at different phases of its life cycle and/or at different positions may result in different elastic modulus values. These undesirable variables can be minimized by indenting on a comparable position which can reflect the entire cell [17], and synchronizing the batch of cells to be tested at a certain stage of the cell cycle such as G0/G1. In this work, we employed an oral cancer cell line UM1, and we took steps to standardize and synchronize the cells, so as to enable comparable conditions for the AFM nanoindentation. The UM1 is a head and neck squamous cell carcinoma (HNSCC) cell line with high metastatic potential [40]. It is used in this work as a prototype of mammalian cancer cells.

\section{Materials and Methods}

\section{Cell culture}

Cells from the oral cancer cell line UM1 were cultured in a DMEM/F12 medium containing $10 \%$ FBS and $1 \%$ penicillin-streptomycin, and were maintained at $37{ }^{\circ} \mathrm{C}$ in a $5 \% \mathrm{CO}_{2}$ incubator (Bionex ${ }^{@}$, Korea). A batch of these cells was prepared for AFM scanning and nucleus staining, by digestion with trypsin in 5 min, followed by harvest and planting onto a $14 \mathrm{~mm}$ diameter glass coverslip (Thermo Menzel, Germany) kept inside a 24-well plate for 24 hours prior to AFM scanning. Another batch of UM1 cells were prepared for AFM nanoindentation by planting onto a $25 \mathrm{~mm}$ diameter glass coverslip which was then placed inside a $35 \mathrm{~mm}$ plastic dish for 24 hours, followed by synchronization into the G0/G1 stage by serum starvation [41-43]. The cells were transferred into a normal DMEM/F12 medium containing 10\% Fetal Bovine Serum in a cell holder maintained by a heater (Biocell ${ }^{\mathrm{TM}}$, JPK) at $37{ }^{\circ} \mathrm{C}$. After these steps, the cells were ready for AFM nanoindentation. 
The batch of cells for AFM structure scanning was treated with the method adopted by Buño [44]. After the treatment, the cells were fixed onto a rough glass coverslip, which was then put inside a Biocell ${ }^{\mathrm{TM}}$ holder which maintained the temperature at $37^{\circ} \mathrm{C}$, and the holder was mounted onto the AFM stage. For cell nucleus staining, the protocol recommended in the product Hoechst 33342 (sigma) was followed. After the staining, the samples were inspected under an integrated microscope with an inverted optical microscope (Nikon Eclipse Ti, Japan) on the top and a Nikon TI-FLC fluorescent exciter at the bottom.

AFM indentation

The cells used in the nanoindentation experiments were living cells in DMEM/F12 medium solution containing 10\% Fetal Bovine Serum maintained at $37^{\mathrm{O}} \mathrm{C}$. These conditions ensure that the cells can survive for at least 4 hours, which is much longer than the entire duration of the indentation tests of not more than 2.5 hours. The AFM employed for cell indentation was a Zeiss microscope integrated with a JPK NanoWizard II controller (JPK instruments AG, Germany). A silicon nitride cantilever-tip (CSG01, NT-MDT) was used, the spring constant of which was calibrated by the thermal fluctuation method [45] to be $0.037 \mathrm{~N} / \mathrm{m}$, and the cantilever sensitivity was calibrated by indenting on glass in the DMEM/F12 medium solution. The vertical deflection of the cantilever as measured by the photodiode and the piezoelectric displacement of the base of the cantilever-tip can therefore be converted directly into force and penetration displacement of the sample's deformation.

To simplify the tip-sample contact geometry [46] and to avoid the AFM tip punching through the very soft cell membrane during indentation, the very end of the CSG01-NT-MDT silicon nitride tip was cut off by focused ion-beam (FIB) milling (FEI Quanta 200 3D FIB/SEM) to form a flat-ended cylinder shape. The FIB process started with rough milling at current $0.5 \mathrm{nA}$, followed by fine milling at $50 \mathrm{pA}$. Figure 1(a,b) shows the resultant AFM tip produced for nanoindentation, the end-diameter of which was measured by the software of FIB instrument to be $1 \mu \mathrm{m}$, length around $5 \mu \mathrm{m}$. A flat-ended tip with a cylindrical shape has the advantage of a constant contact area with the sample during indentation. Therefore accurate determination of the solid contact point between the tip and sample is not necessary in our measurements, and this greatly simplifies the analysis.

The AFM used in the present nanoindentation experiments has the feature that the sample sits on a stationary platform while the motion of the clamping base of the cantilever-tip is controlled (Figure 1c). Thus, during indentation, the height $\delta$ of the cantilever base was programmed to move downward, and the deflection of the cantilever's end was detected by the photodiode as the output. With the datum set at the contact point with the sample, the deflection of the cantilever's end as detected by the photodiode is simply the indentation depth $h$. 
The common Hertzian indentation protocol, as described above, was implemented on top of the nuclei of the cells at different displacement rates of the AFM cantilever base (i.e. $\delta$ ) of $0.1 \mu \mathrm{m} / \mathrm{s}, 0.275 \mu \mathrm{m} / \mathrm{s}, 0.55 \mu \mathrm{m} / \mathrm{s}$ or $1.1 \mu \mathrm{m} / \mathrm{s}$ in different tests. The detailed load profile was as follows: the tip was first approached, followed by retraction for $5 \mu \mathrm{m}$ in 1 second, then indentation to a depth of $5.5 \mu \mathrm{m}$ according to the speed of the AFM cantilever base adopted, then holding for 30 seconds and finally retraction at $0.1 \mu \mathrm{m} / \mathrm{s}$ for 10 seconds. The Young modulus $E$ was then calculated by Sneddon's formula for flat-punch indentation [47] which originates from the Hertzian contact model:

$$
P=2 a\left(\frac{E}{1-v^{2}}\right) h
$$

where $P$ is the indentation force, $E$ is Young's modulus, $v$ is the Poisson ratio which is assumed to be 0.5 (i.e. incompressibility assumed) for the cell samples, $a(=0.5$ $\mu \mathrm{m}$, see Figure $1(\mathrm{a}, \mathrm{b}))$ is the end-radius of the cylindrical flat-end tip, and $h$ is the indentation depth. From Figure 1c, the indentation force is given by $P=k(\delta-h)$.

For the rate-jump method, the experimental procedure is similar to the above for the Hertzian protocol except for the use of the same loading displacement rate of 0.55 $\mu \mathrm{m} / \mathrm{s}$ followed by holding of 30s, followed by unloading at a displacement rate of 0.2 $\mu \mathrm{m} / \mathrm{s}, 0.1 \mu \mathrm{m} / \mathrm{s}$ or $0.067 \mu \mathrm{m} / \mathrm{s}$ in different tests. The Young modulus was calculated by the following formula [31-32]:

$$
\Delta \dot{P}=2 a\left(\frac{E}{1-v^{2}}\right) \Delta \tilde{h}
$$

where $\Delta \vec{P}$ is the resultant jump in the load rate at the onset of the unloading, i.e. $\Delta \dot{P}=\dot{P}_{+}-\dot{P}_{-}$where $\dot{P}_{-}$and $\dot{P}_{+}$are the load rates before and after the jump at the onset of the unloading, and $\Delta \tilde{h}^{\prime} \tilde{h}_{+}-\tilde{h}_{-}$is the jump of the indentation depth rate where $\dot{h}_{-}$and $\dot{h}_{+}$are the indentation depth rates just before and after the jump at the onset of unloading. The $a, E$ and $v$ in eqn. (2) have the same meanings as in eqn. (1). The similar forms between eqns. (1) and (2) in fact stem from a theorem derived in ref. [32], which states that the effective elastic modulus for a viscoelastic material is given by solving the stress-strain problem for the same spatial domain but treating 
the material as if it were purely elastic, and then substituting force and displacement in the resultant relation by their rate jumps respectively. The $E /\left(1-v^{2}\right)$ obtained from eqn. (2) is in fact the resultant value of all the spring elements in the viscoelastic constitutive relation with all the dashpots (assumed nonlinear in general) ignored, and hence should be an intrinsic property of the specimen. From Figure 1c, $\Delta \vec{P}$ in eqn. (2)

is given as $\Delta \dot{P}=k(\Delta \hat{\delta}-\Delta \dot{h})$.

The indentation was always made on the most bulging part of the cell shown under AFM, and normally this was also the center of the cell. Each cell was indented only once by either the rate-jump or Hertz protocol, and for a given loading profile, more than 20 repeated measurements were made and each of these was on a different cell.

\section{Results}

\section{Cell observation}

It has been reported that stiffness can be highly variant by indenting on different parts of the cell [5, 48-51], and thus randomly indenting on the rim or bulging parts of the cells should lead to large scatter of the results. Figure 2a shows the AFM scanned morphology of a typical UM1 cell, where the nucleus makes up a big area inside the cell. In the fluorescent microscopy image in Figure 2b, the brightest and oval-shaped parts are the nuclei stained by Hoechst. The nucleus is not always close to the center of cells, and Figure 2a in fact shows that the nucleus is located near the rim of that scanned cell. Thus, when performing indentation, the tip were made to indent on the most bulging part of the cells (the arrows shown in Figure 2c), which should be the exact location of the nucleus.

\section{AFM nanoindentation}

Figure 3a shows typical indentation force $P$ vs indentation depth $h$ curves for the UM1 cell line at different loading rates using the Hertzian indentation protocol. According to eqn. (1), the average slope of such a curve should be proportional to the elastic modulus, but it can be seen from Figure 3a that the average slope increases with the loading rate. This is a typical viscoelastic response. Figure 3(b,c) show the elastic modulus calculated using eqn. (1) at different loading rates - the calculation was actually performed by the JPK DP data processing software. In Figure 3(b), only data for shallow indentation depths up to $100 \mathrm{~nm}$ were used, and in Figure 3(c), data up to 500nm were used, in accordance with the usual practice [52-55]. The apparent 
elastic modulus here clearly shows a steady rise with the loading rate, in agreement with Li et al.'s observation from breast cancer cells [16].

Figure 4a shows plots of the height $\delta$ of the base of the cantilever vs time as input conditions in the rate-jump protocol, in which the loading history was programmed to be identical up to the end of the hold at 41s, followed by different retraction rates in different tests. At the start of each experiment, the tip approaching protocol of the machine sensed the sample's surface by making the tip contact the sample until a small contact force of about $2.6 \mathrm{nN}$ was reached, followed by retracting the tip slightly until the contact force was slightly negative in the attractive regime. The state of the machine at the end of this procedure is known as the set point, and in the loading profiles shown in Figure 4a, the displacement $\delta$ of the AFM cantilever base (see Figure 1c) is relative to this set point, i.e. zero marks the set point, $\delta>0$ marks downward travel and vice versa. After the set point was detected, the cantilever base was programmed to move upward (i.e. $\delta$ becoming $<0$ ) by $5 \mu \mathrm{m}$ at a constant speed, hence retracting the tip further from the sample. After this, the cantilever base was brought down (i.e. $\delta$ increasing towards $>0$ ) at a constant speed as shown in Figure 4a to make the tip indent on the sample, and the programmed travel of the cantilever base past the set point in the indentation direction was $500 \mathrm{~nm}$. Followed by a hold period of $30 \mathrm{sec}$, the cantilever base was made to retract at different speeds of 0.067, 0.1 and $0.2 \mu \mathrm{m} / \mathrm{s}$ as shown in Figure 4a. Figure 4(b,c) show the corresponding plots of the indentation force vs time, and the displacement $h$ of the end of the AFM cantilever as detected by the photo-diode (see Figure 1c) vs time, respectively. It is interesting to see from Figure $4 \mathrm{~b}$ that during the hold period, the indentation force dropped from 10, 13 or $16 \mathrm{nN}$ in the three different curves to about $4 \mathrm{nN}$, and this is manifestation of viscous relaxation of the cells. Plots such as Figures 4(b,c) were used to obtain the rate jumps $\Delta \dot{P}$ and $\Delta \dot{h}$ in eqn. (2), and Figure 4d and Table 1 show the calculated Young's modulus. One-way ANOVA analysis of these data obtained from the rate-jump protocol showed that the mean values of the modulus at different retraction rates are not significantly different $(\mathrm{p}>0.05)$. However, ANOVA analysis showed that the mean values of the Young modulus obtained from the Hertzian protocol are significantly different $(\mathrm{p}<0.001)$ between the different loading rates. It should be noted that in both the Hertzian protocol and the rate-jump protocol, the maximum indentation depth was on the order of $1 \mu \mathrm{m}$. For the Hertzian protocol, the modulus was calculated using data up to indentation depths of $100 \mathrm{~nm}$ and 500nm, and although these two sets of data differ, they both exhibit a rising trend with the loading rate as shown in Figure 3(b,c) and Table 1. 


\section{Discussion}

While our earlier treatment deals with viscoelastic behavior [31,32], the mechanical response of the cytoplasm-membrane-cytoskeleton structure of a cell may be better described as poro-viscoelastic [56, 57], where the coupling between the stresses in the solid components (i.e. cytoskeleton and cell membrane) and the pressure in the fluid component (i.e. cytoplasm) is considered. A very general poro-viscoelastic constitutive model may be a general network composing (i) linear elastic elements of the form

$$
\sigma_{i j}=c_{i j k l} \varepsilon_{k l}+\delta_{i j} \lambda^{p}
$$

and (ii) viscous dashpot elements such as, but not restricted to, the Odqvist form [32]

$$
\dot{\varepsilon}_{i j}=\frac{3}{2} c \sigma_{e}^{m-1} S_{i j} .
$$

Here, $\sigma_{k l}$ and $\varepsilon_{i j}$ are the stress and strain tensors, $\varepsilon_{i j}=\left(u_{i, j}+u_{j, i}\right) / 2$, where $u_{i}$ is the displacement, and ()$=\partial() / \partial t$ denotes time rates. In eqn. (3a), $c_{i j k l}$ are the elastic constants of the solid components, $\lambda^{p}$ is the eigenstress due to the fluid, and $\delta_{i j}$ is the Kronecker delta. In eqn. (3b),

$$
\sigma_{e}=\sqrt{\sigma_{11}^{2}+\sigma_{22}^{2}+\sigma_{33}^{2}-\sigma_{11} \sigma_{22}-\sigma_{22} \sigma_{33}-\sigma_{11} \sigma_{33}+3\left(\sigma_{12}^{2}+\sigma_{23}^{2}+\sigma_{31}^{2}\right)}
$$

is an effective stress, and $s_{i j}=\sigma_{i j}-\left(\delta_{i j} / 3\right) \sigma_{k k}$ is the deviatoric stress, and $c$ and $m$ are constants. Such a form of poro-viscoelastic constitutive law is similar to that developed recently by Suvorov and Selvadurai [56], but is more universal in the sense that (i) the elasticity as described by eqn. (3a) is anisotropic, and (ii) the viscous components as described by eqn. (3b) are in general non-linear. The spring elements in eqn. (3a) and dashpot elements in eqn. (3b) can be arranged in any network arrangement as appropriate to the cell structure, including, but not limited to, the standard linear solid model.

Following our earlier approach [31, 32], we are interested in the response due to a step change in the loading rate or in the displacement rate at time $t_{c}$, which results in step changes $\Delta \dot{\sigma}_{i j}$ in the stress field and $\Delta \dot{\varepsilon}_{i j}$ in the strain-rate field across $t_{c}$, after 
settlement of any transient effects. From eqns. (3a) and (3b), these step changes are given by

$$
\Delta \dot{\sigma}_{i j}=c_{i j k l} \Delta \dot{\varepsilon}_{k l}+\delta_{i j} \Delta \dot{\lambda}^{p}
$$

for the elastic elements, and

$$
\Delta \dot{\varepsilon}_{i j}=\frac{3}{2} c\left[\left.\left(\sigma_{e}^{m-1} s_{i j}\right)\right|_{+}-\left.\left(\sigma_{e}^{m-1} s_{i j}\right)\right|_{-}\right]=0
$$

for the dashpot elements. The last step in eqn. (4b) stems from the fact that stresses in the system must be continuous across the jump. In eqn. (4a), $\Delta \dot{\lambda}^{p}$ is the jump of the rate of the eigenstress on the solid skeleton due to the fluid. The eigenstress is proportional to the fluid pressure $p$; for example, Suvorov and Selvadurai showed that for an isotropic material, $\lambda^{p}=-\left(1-K_{D} / K_{S}\right) p$, where $K_{D}$ is the bulk modulus of the drained solid structure and $K_{S}$ is the bulk modulus of the solid phase itself. In a cell during nanoindentation, as long as the membrane is not broken, the fluid content inside is conserved and so to a good approximation, the fluid pressure $p$ is conserved for a short duration before and after the rate jump across $t_{c}$. Thus, $\Delta \dot{p}=0$ and $\Delta \dot{\lambda}^{p}=0$ across the jump, and so eqn. (4a) reduces to

$$
\Delta \dot{\sigma}_{i j}=c_{i j k l} \Delta \dot{\varepsilon}_{k l} .
$$

Together with eqn. (4b), eqn. (4c) indicates that $\left\{\Delta \dot{u}_{i}, \Delta \dot{\varepsilon}_{i j}, \Delta \dot{\sigma}_{i j}\right\}$ can be solved as the \{displacement, strain, stress $\}$ of a linear elastic problem, defined on the same space domain and described by the same elastic constants $c_{i j k l}$ as the original poro-viscoelastic material. For this reduced problem, due to eqn. (4b), the model network consists of only the elastic elements which are the same as the original poro-viscoelastic material, without the dashpots.

The analysis here therefore explains why even with the fluid content and the viscous factors in the cell, a valid solution in eqn. (2) for the cell, which is supposed to be poro-viscoelastic, can be obtained by replacing $P$ and $h$ in the elastic solution in eqn. (1) by $\Delta \dot{P}$ and $\Delta \dot{h}$ respectively. The results in Table 1 show that the rate-jump protocol involving eqn. (2) yields invariant elastic modulus for the UM1 oral cancer cell line, while the Hertzian protocol involving eqn. (1) does not. It is important to 
realize that our treatment here assumes eqns. (3a) and (3b) to hold within a short time window $\left(t_{c}-\delta t, t_{c}+\delta t\right)$ at about $t_{c}$, which is the moment the step change is applied. The step changes given in eqns. $4(a-c)$ are the result of writing down eqns. 3(a,b) at the beginning and end of this time window, during which transients may occur, and subtracting between these two time points. In other words, the elastic constant measured, which is the resultant of $c_{i j k l}$ in the viscoelastic network, is the value reflecting the sample's microstructure at time $t_{c}$ - this microstructure should in general depend on the treatment history of the sample up to $t_{c}$, but as long as this history up to $t_{c}$ is the same, this will be a constant factor. In the present rate-jump experiments, the load history up to the onset of the unload was indeed the same, and only the retraction rate immediately after the onset of the unload was different between the tests (c.f. Figure 4a). The elastic modulus obtained by eqn. (2) therefore represents the state of the specimen at the point of the onset of the unload, and so we should expect the states of the cells tested here to be comparable at the onset of the unload: the cells were synchronized at the G0/G1 phase for the indentation tests, and the load history was identical up to the onset of unload. Indeed the elastic modulus measured at the onset of unload was shown to be invariant with respect to the retraction rate, and so the rate-jump protocol is verified for this cell line. On the other hand, for the Hertzian protocol, Figure 3(b,c) and Table 1 show that the evaluated elastic modulus changes with the loading rate as well as the indentation depth range used in the calculation. In fact, the Hertzian protocol assumes that the cell remains elastic over the whole depth range used in the calculation, and so the modulus data calculated from data up to large indentation depths such as $500 \mathrm{~nm}$ should be rather unreliable.

Although the rate-jump protocol has been found valid for synthetic materials including soft metals and polymers in different test geometries [29-32], this is the first time this protocol is verified for living cells. This shows that the present cell line can be fairly accurately described as viscoelastic or poro-viscoelastic in terms of their deformation under load. Moreover, the results indicate that the rate-jump protocol is better than the conventional Hertzian protocol for measuring stiffness of cells, since the unwanted effects of viscosity are eliminated and the measured stiffness becomes an intrinsic property of the cell independent of the way in which the experiment is carried out. The present work also shows the importance to synchronize the cells before indentation, and the merit brought about by adopting a flat-ended geometry of the indenter tip. The rate-jump protocol should form a basis of standardization where results of the same cell type can be compared between laboratories.

\section{Conclusion}


The commonly used Hertzian protocol for nanoindentation yields apparent elastic modulus of UM1 cells that increases with the loading rate, indicating strong effects of viscoelasticity. However, a rate-jump protocol developed for viscoelastic materials gives invariant elastic modulus. The applicability of this rate-jump protocol to poro-viscoelastic materials is also demonstrated theoretically.

\section{Acknowledgements}

The work done in this article was supported by a grant from the University Grants Committee of the Hong Kong Special Administration Region, P.R. China (Project No.SEG-HKU06).

\section{References}

[1] K.A. Ward, W.I. Li, S. Zimmer, T. Davis, Viscoelastic properties of transformed cells: role in tumor cell progression and metastasis formation, Biorheology 28 (3-4) (1991) 301-313.

[2] S. Suresh, J. Spatz, J.P. Mills, A. Micoulet, M. Dao, C.T. Lim, M. Beil, T. Seufferlein, Connections between single-cell biomechanics and human disease states: Gastrointestinal cancer and malaria, Acta Biomaterialia. 1 (2005)15-30.

[3] J. Guck, S. Schinkinger, B. Lincoln, F. Wottawah, S. Ebert, M. Romeyke, D. Lenz, H.M. Erickson, R. Ananthakrishnan, D. Mitchell, J. Kas, S. Ulvick, C. Bilby, Optical deformability as an inherent cell marker for testing malignant transformation and metastatic competence, Biophys. J. 88(2005)3689-3698.

[4] Suresh S, Elastic clues in cancer detection, Nat. Nanotechnol. 2(2007)748-749.

[5] S.E. Cross, Y.S. Jin, J. Rao, J.K. Gimzewski, Nanomechanical analysis of cells from cancer patients, Nat. Nano. 2 (12) (2007) 780-783.

[6] E.M. Darling, S. Zauscher, J.A. Block, F. Guilak, Thin-Layer Model for Viscoelastic, Stress-Relaxation Testing of Cells Using Atomic Force Microscopy: Do Cell Properties Reflect Metastatic Potential? Biophys. J. 92(5) (2007)1784-1791.

[7] S.E. Cross, S.J. Yu, J. Tondre, R. Wong, J.Y. Rao, J.K. Gimzewski, AFM-based analysis of human metastatic cancer cells, Nanotechnology. 19 (2008) 384003

[8] V. Swaminathan, K. Mythreye, E.T. O’Brien, A. Berchuck, G.C. Blobe, R. Superfine, Mechanical Stiffness Grades Metastatic Potential in Patient Tumor Cells and in Cancer Cell Lines, Cancer Res.71(15) (2011)1-6.

[9] M. Lekka, P. Laidler, D. Gil, J. Lekki, Z. Stachura, A.Z. Hrynkiewicz, Elasticity of normal and cancerous human bladder cells studied by scanning force microscopy, Eur. Biophys. J. 28 (4) (1999) 312-316.

[10]M. Lekka, J. Lekki, M. Marszalek, P. Golonka, P. Stachura, B. Cleff, A.Z. Hrynkiewicz, Local elastic properties of cells studied by SFM, Appl Surf Sci. 
141(1999)345-50.

[11]M. Lekka, P. Laidler, J. Ignacak, M. Labedz, J. Lekki, H. Struszczyk, Z. Stachura, A.Z. Hrynkiewicz, The effect of chitosan on stiffness and glycolytic activity of human bladder cells, Biochim. Biophys. Acta 1540 (2) (2001) 127-136.

[12]S. Park, D. Koch, R. Cardenas, J. Kas, C.K. Shih, Cell motility and local viscoelasticity of fibroblasts, Biophys. J. 89 (6) (2005) 4330-4342.

[13]M.J. Rosenbluth, W.A. Lam, D.A. Fletcher, Force microscopy of nonadherent cells: a comparison of leukemia cell deformability, Biophys J. 90(2006)2994-3003.

[14]W.A. Lam, M.J. Rosenbluth, D.A. Fletcher, Chemotherapy exposure increases leukemia cell stiffness, Blood. 109(2007)3503-3508.

[15]S. Suresh, Biomechanics and biophysics of cancer cells, Acta Mater. 55 (12) (2007) 3989-4014.

[16]Q.S. Li, G.Y.H. Lee, C.N. Ong, C.T. Lim, AFM indentation study of breast cancer cells. Biochemical and Biophysical Research Communications, 374 (2008) 609-613.

[17]M. Lekka, P. Laidler, Applicability of AFM in cancer detection, Nature Nanotechnology. 4(2009)72-73.

[18]S. Iyer, R.M. Gaikwad, V. Subba-Rao, C.D. Woodworth, Igor Sokolov, Atomic force microscopy detects differences in the surface brush of normal and cancerous cells, Nature Nanotechnology. 4(2009)389 - 393.

[19]R. Hochmuth, Micropipette aspiration of living cells, J. Biomech. 33(2000) $15-22$.

[20]G. Zhang, M. Long, Z.Z. Wu, W.Q. Yu, Mechanical properties of hepatocellular carcinoma cells, World J Gastroenterol. 8(2002)243-246.

[21]W. Yao, L. Gu, D. Sun, W. Ka, Z. Wen, S. Chien. Wild type p53 gene causes reorganization of cytoskeleton and therefore the impaired deformability and difficult migration of murine erythroleukemia cells. Cell Motil Cytoskeleton 56(2003)1-12.

[22]K. Chen, D. Li, Y.H. Jiang, W.J. Yao, X. J. Wang, X.C. Wei, J. GAO, L.D. Xie, Z.Y. Yan, Z.Y. Wen, S. Chien. Influence of expressed TRAIL on biophysical properties of human leukemic cell line JURKAT, Cell Res. 14(2004)161-168.

[23]T. Korb, K. Schluter, A. Enns, H.U. Spiegel, N. Seninger, G.L. Nicolson, J. Haier, Integrity of actin stress fibers and microtubules influences metastatic tumor cell adhesion, Exp Cell Res. 299(2004)236-47

[24]W. Saadi, S.J. Wang, F. Lin, N.L. Jeon, A parallel-gradient microfluidic chamber for quantitative analysis of breast cancer cell chemotaxis, Biomed Microdevices. 8(2) (2006)109-118.

[25]N. Bao, Y.H. Zhan, C. Lu, Microfluidic Electroporative Flow Cytometry for Studying Single-Cell Biomechanics, Anal. Chem. 80(2008)7714-7719.

[26]J.W. Song, S.P. Cavnar, A.C. Walker, K.E. Luker, M. Gupta, Y.C. Tung, G.D. Luker, S. Takayama, Microfluidic endothelium for studying the intravascular adhesion of metastatic breast cancer cells, PLoS One. 4(6) (2009) e5756.

[27]H. Hertz, Über die Berührung fester elastischer Körper, J. Reine Angew Math. 92 (1882)156-171. 
[28]Y.L. Chan, A.H.W. Ngan, Invariant elastic modulus of viscoelastic materials measured by rate-jump tests, Polymer testing. 29(2010)558-564.

[29]A.H.W. Ngan, B. Tang, Viscoelastic effects during unloading in depth-sensing indentation, J. Mater. Res. 17(10) (2002)2604-2610.

[30]B. Tang, A.H.W. Ngan, Accurate measurement of tip-sample contact size during nanoindentation of viscoelastic materials, Journal of Materials Research. 18(5) (2003) 1141-1148.

[31]B. Tang, A.H.W. Ngan, Nanoindentation using Atomic Force Microscope, Phil. Mag. 91 (2011) 1329-1338.

[32]A.H.W. Ngan, B. Tang, Response of power-law-viscoelastic and time-dependent materials to rate jumps, J. Mater. Res. 249(3) (2009)853-862.

[33]A. Daniel, R. Fletche, D. Mullins, Cell mechanics and the cytoskeleton, Nature. 463(2010) 485-492.

[34]M.F. Olson, E. Sahai, The actin cytoskeleton in cancer cell motility, Clin Exp Metastasis. 26(2009)273-287.

[35]H. Alan, The cytoskeleton and cancer, Cancer Metastasis Rev. 28 (2009) 5-14.

[36]D. Yamazaki, S. Kurisu, T. Takenawa, Regulation of cancer cell motility through actin reorganization, Cancer Sci. 96 (2005) 379-386.

[37]C. Rotsch, K. Jacobson, M. Radmacher, Dimensional and mechanical dynamics of active and stable edges in motile fibroblasts investigated by using atomic force microscopy, Proc. Natl. Acad. Sci. USA. 96 (3) (1999) 921-926.

[38]E. Henderson, P.G. Haydon, D.S. Sakaguchi, Actin filament dynamics in living glial cells imaged by atomic force microscopy, Science. 257(5078) (1992)1944-1946.

[39]K.B hadriraju, L.K. Hansen, Extracellular matrix- and cytoskeleton-dependent changes in cell shape and stiffness, Exp. Cell Res. 278(2002) 92-100.

[40]S. Nakayama, A. Sasaki, H. Mese, R.E. Alcalde, T. Matsumura, Establishment of high and low metastasis cell lines derived from a human tongue squamous cell carcinoma, Invision Metastasis. 18(5-6) (1998-1999)219-228.

[41]P.L. Puri, C. Balsano, V.L. Purgio, P. Chirillo, G. Natoli, L. Ricci, E. Mattei. A. Graessmann, M. Levrero, MyoD prevents cyclin A-CDK2-containing E2F complexes formation in terminally differentiated myocytes, Oncogene. 14(1997)1171-1184.

[42]J. Tesfaigzi, D.M. Carlson, Cell cycle-specific expression of G (0) SPR1 in Chinese hamster ovary cells, Exp. Cell Res. 228(1996)227-282.

[43]G.S. Dbaibo, M.Y. Pushkareva, S. Jayadev, J.K. Schwarz, J.M. Horowitz, L.M. Obeid, Y.A. Hannun, Retinoblastoma gene product as a downstream target for a ceramide-dependent pathway of growth arrest, Proc. Natl. Acad. Sci. U.S.A. 92(1995)1347-1351.

[44]I. Buño, A. Juarranz, M. Cañete, A. Villanueva, J. Gosalvez, J.C. Stockert, Image Processing and Analysis of Fluorescent Labelled Cytoskeleton, Micron. 29(6) (1998)445-449.

[45]J.L. Hutter, J. Bechhoefer, Calibration of atomic-force microscope tips, Rev. Sci. Instrum. 64(1993)1868-1873.

[46]G. Moeller, AFM Nanoindentation of Viscoelastic Materials with Large 
End-Radius Probes, Journal of Polymer Science Part B-Polymer Physics, 47(16) (2009) 1573-1587.

[47]I.N. Sneddon, The relation between load and penetration in the axisymmetric boussinesq problem for a punch of arbitrary profile, Int. J. Eng. Sci. 3(1) (1965) 47-57.

[48]S.G. Shroff, D.R. Saner, R. Lal, Dynamic micromechanical properties of cultured rat atrial myocytes measured by atomic force microscopy, Am. J. Physiol. Cell Physiol. 269(1995)286-292.

[49]U.G. Hofmann, C. Rotsch, W.J. Parak, M. Radmacher, Investigating the cytoskeleton of chicken cardiocytes with the atomic force microscope, J. Struct. Biol. 119(1997)84-91.

[50]A.B. Mathur, G.A. Truskey, W.M. Reichert, Atomic force and total internal reflection fluorescence microscopy for the study of force transmission in endothelial cells. Biophys J. 78(2000)1725-1735.

[51]L. Sirghi, J. Ponti, F. Broggi, F. Rossi, Probing elasticity and adhesion of live cells by atomic force.microscopy indentation, Eur Biophys J. 37 (2008)935-945.

[52]G. Persch, C. Born, B. Utesch, Nano-hardness investigations of thin films by an atomic force microscope, Microelec. Eng. 24(1994)113-121.

[53]M. Stolz, R. Raiteri, A.U. Daniels, M.R. VanLandingham, W. Baschong, U. Aebi, Dynamic Elastic Modulus of Porcine Articular Cartilage Determined at Two Different Levels of Tissue Organization by Indentation-Type Atomic Force Microscopy, Biophys J. 86(5) (2004)3269-3283.

[54]T.G. Kuznetsova, M.N. Starodubtseva, N.I. Yegorenkov, S.A. Chizhik, R.I. Zhdanov, Atomic force microscopy probing of cell elasticity, Micron 38 (8) (2007) 824-833.

[55]A.E. Pelling, D.W. Dawson, D.M. Carreon, J.J. Christiansen, R.R. Shen, M.A. Teitell, J.K. Gimzewski, Distinct contributions of microtubule subtypes to cell membrane shape and stability / Nanomedicine: Nanotechnology, Biology, and Medicine 3 (2007) 43-52.

[56]A.P. Suvorov, A.P.S. Selvadurai, Macroscopic constitutive equations of thermo-poroviscoelasticity derived using eigenstrains, Journal of the Mechanics and Physics of Solids, 58 (2010) 1461-1473.

[57] M. Calli, E. Fornasiere, J. Cugnoni, M.L. Oyen, Poroviscoelastic characterization of particle-reinforced gelatin gels using indentation and homogenization, Journal of the Mechanical Behavior of Biomedical Materials 4 (2011) 610-617. 


\section{Figure Captions}

Fig.1a: End-on view of silicon nitride tip produced by FIB milling.

Fig.1b: Side view of silicon nitride tip produced by FIB milling.

Fig.1c: Schematic showing the AFM used.

Fig.2a: AFM scanned morphology of a UM1 cell.

Fig.2b: Nucleus of UM1 stained by Heochst under fluorescent microscope.

Fig.2c: UM1 cells indented by AFM tip, top view under optical microscope.

Fig.3a: Indentation force vs indentation depth curve for UM1 cells at different loading rates using the Hertzian indentation protocol.

Fig.3(b,c): Apparent elastic modulus of UM1 cell line at different loading rates calculated with the Hertzian indentation protocol using data up to (b) $100 \mathrm{~nm}$ and (c) $500 \mathrm{~nm}$ of indentation depth.

Fig.4a: Displacement of cantilever base vs time curve used in rate jump tests on UM1 cell line. Here, zero height is the set point detected by the machine, $\delta>0$ means downward travel of the cantilever base, and vice versa. The load history is identical up to the end of the hold at $41 \mathrm{~s}$, followed by retraction at rates of $0.2 \mu \mathrm{m} / \mathrm{s}, 0.1 \mu \mathrm{m} / \mathrm{s}$ and $0.067 \mu \mathrm{m} / \mathrm{s}$ respectively.

Fig.4b: Resultant indentation force $P$ vs time curve in rate jump tests using the loading profiles in Fig. 4a.

Fig.4c: Resultant displacement $h$ of end of cantilever as detected by the photo-diode vs time in rate jump tests using the loading profiles in Fig. 4a. Zero marks the set point, $h>0$ means downward movement of the tip, and vice versa.

Fig.4d: The elastic modulus of UM1 cell line with different unloading rates in the rate-jump method. 
Table1: Comparison of the elastic modulus (average \pm SD) of UM1 cell line between Hertzian model and rate-jump method (unit: $\mathrm{kPa} ; \mathrm{n}=20$ )

\begin{tabular}{|c|c|c|c|c|c|}
\hline \multirow{3}{*}{$\begin{array}{c}\text { Hertzian } \\
\text { model }\end{array}$} & Loading rate & $0.1 \mu \mathrm{m} / \mathrm{s}$ & $0.275 \mu \mathrm{m} / \mathrm{s}$ & $0.55 \mu \mathrm{m} / \mathrm{s}$ & $1.1 \mu \mathrm{m} / \mathrm{s}$ \\
\cline { 2 - 6 } & Modulus up to $100 \mathrm{~nm}$ & $0.7 \pm 0.31$ & $1.18 \pm 0.55$ & $1.56 \pm 0.64$ & $1.91 \pm 0.7$ \\
\cline { 2 - 6 } & Modulus up to 500nm & $1.92 \pm 0.82$ & $3.9 \pm 0.88$ & $5.85 \pm 1.56$ & $8.46 \pm 3.63$ \\
\hline \multirow{2}{*}{ Rate-jump } & Unloading rate & $0.2 \mu \mathrm{m} / \mathrm{s}$ & $0.1 \mu \mathrm{m} / \mathrm{s}$ & \multicolumn{2}{|c|}{$0.067 \mu \mathrm{m} / \mathrm{s}$} \\
\cline { 2 - 6 } & Modulus at $~ 500 \mathrm{~nm}$ & $3.65 \pm 1.82$ & $4.03 \pm 1.81$ & \multicolumn{2}{|c|}{$3.49 \pm 1.64$} \\
\hline
\end{tabular}




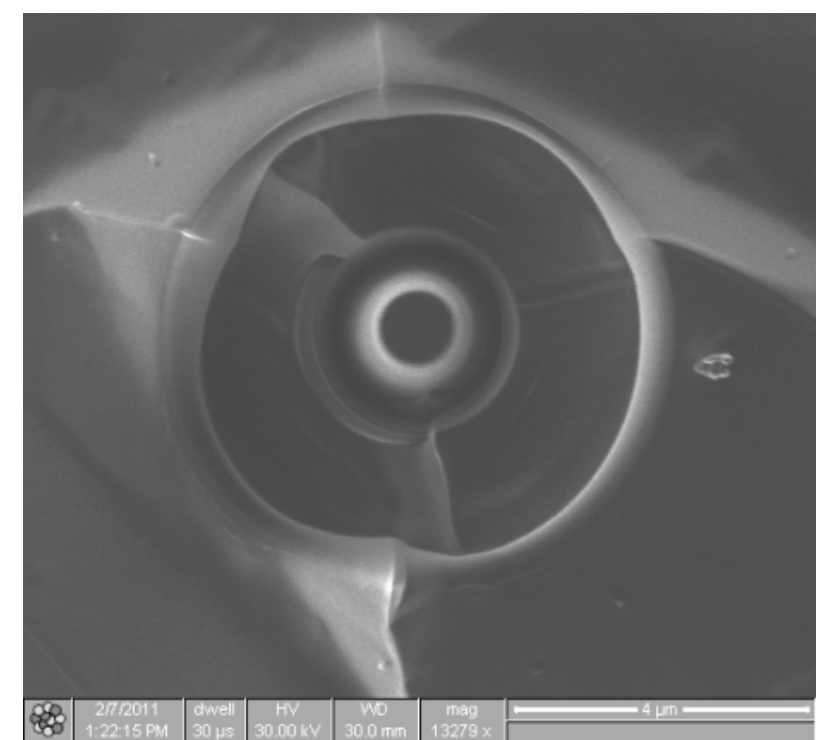

Fig.1a: End-on view of silicon nitride tip produced by FIB milling

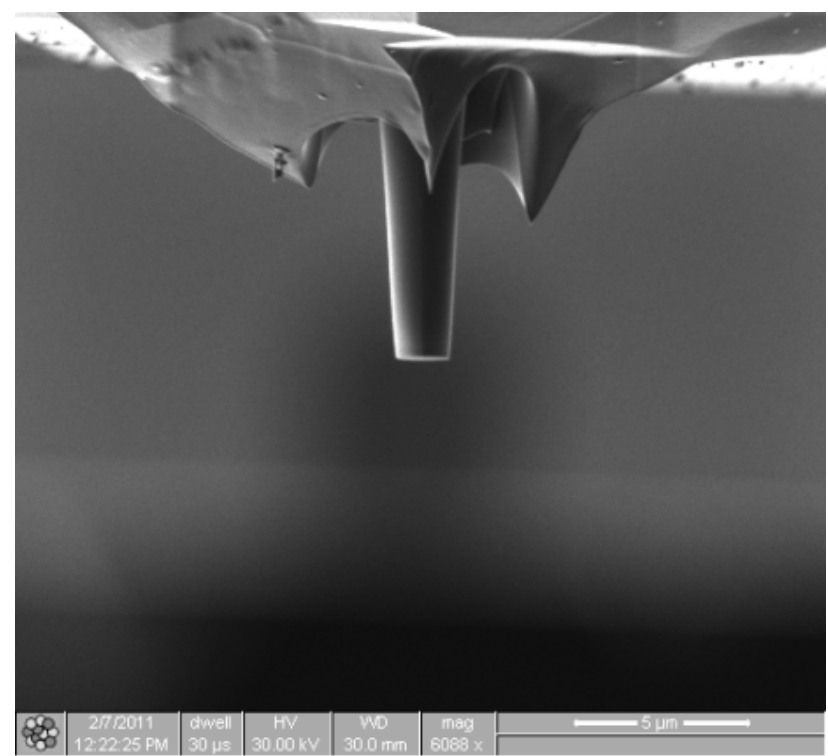

Fig.1b: Side view of silicon nitride tip produced by FIB milling 


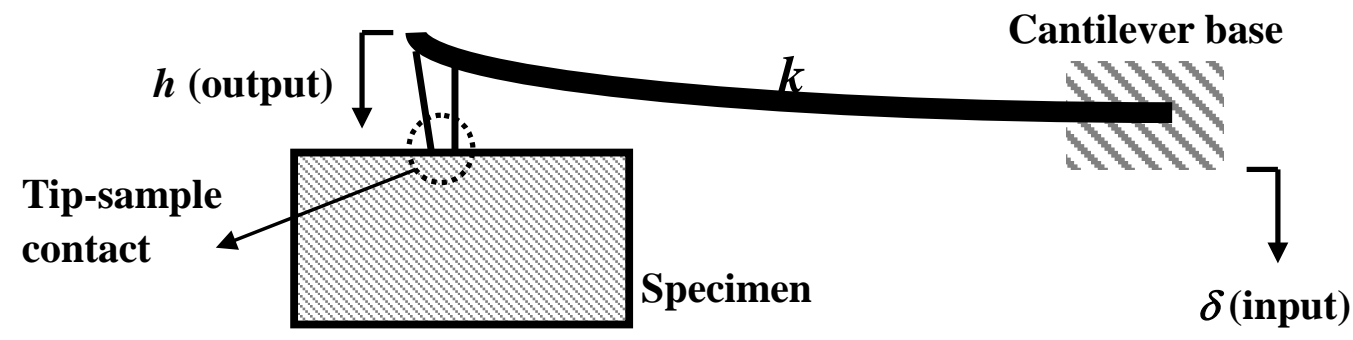

Fig.1c: Schematic showing the AFM used 


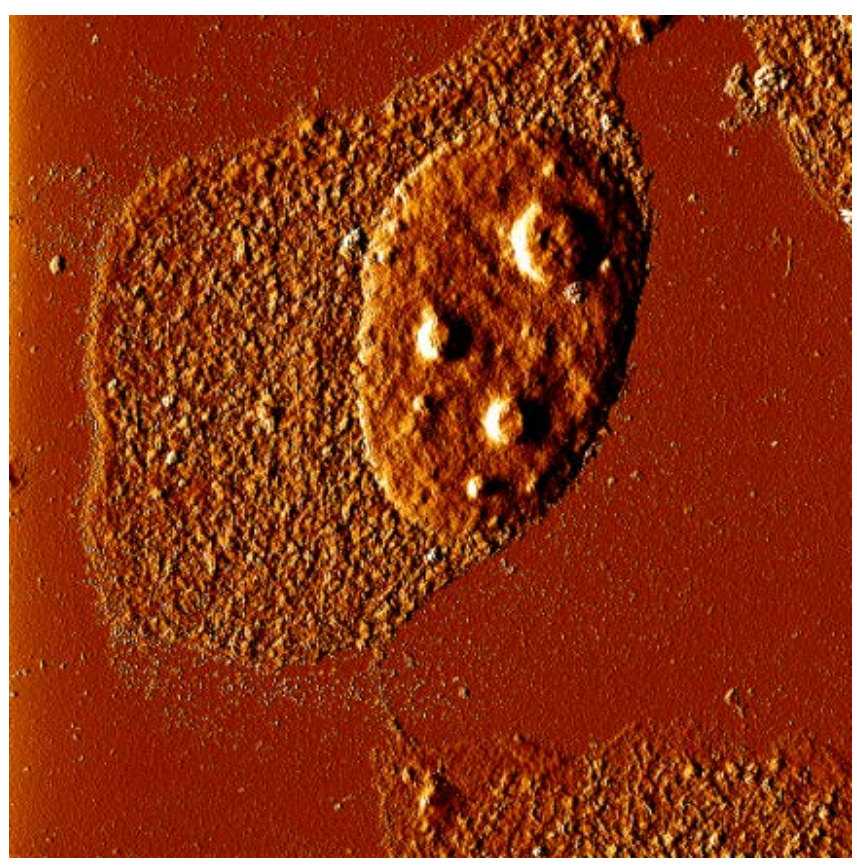

Fig.2a: AFM scanned morphology of a UM1 cell

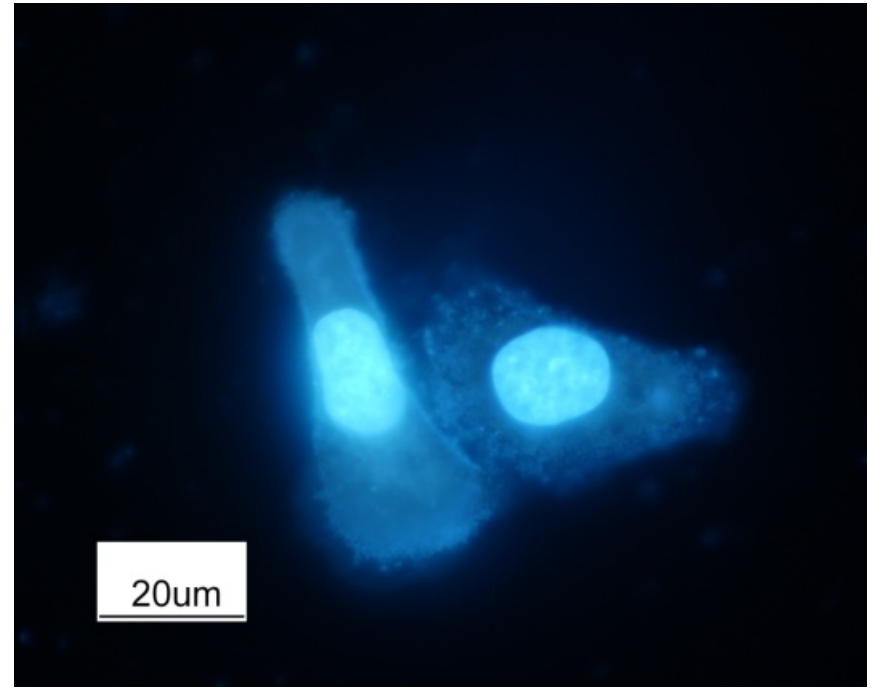

Fig.2b: Nucleus of UM1 stained by Heochst under fluorescent microscope 


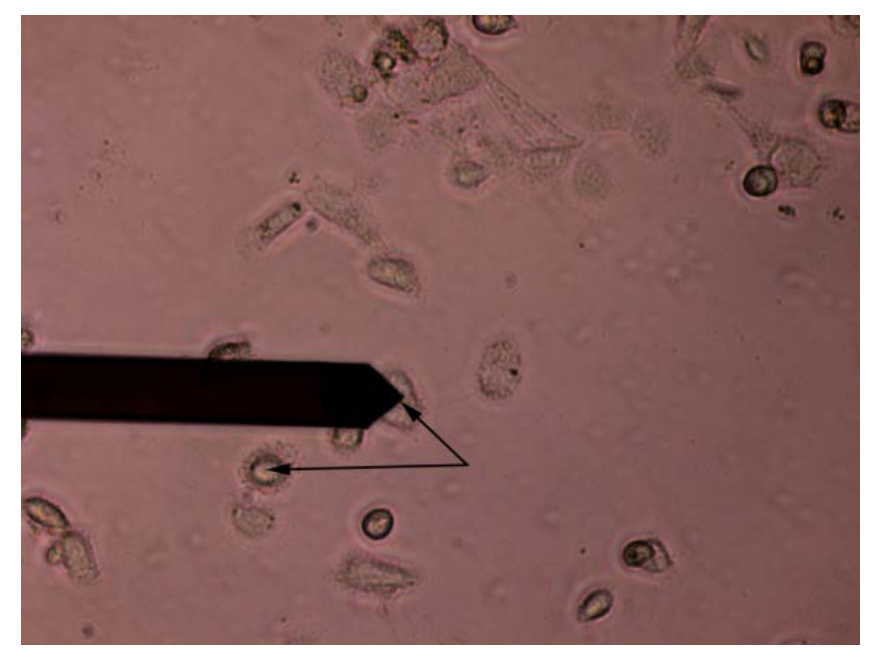

Fig.2c: UM1 cells indented by AFM tip, top view under optical microscope 


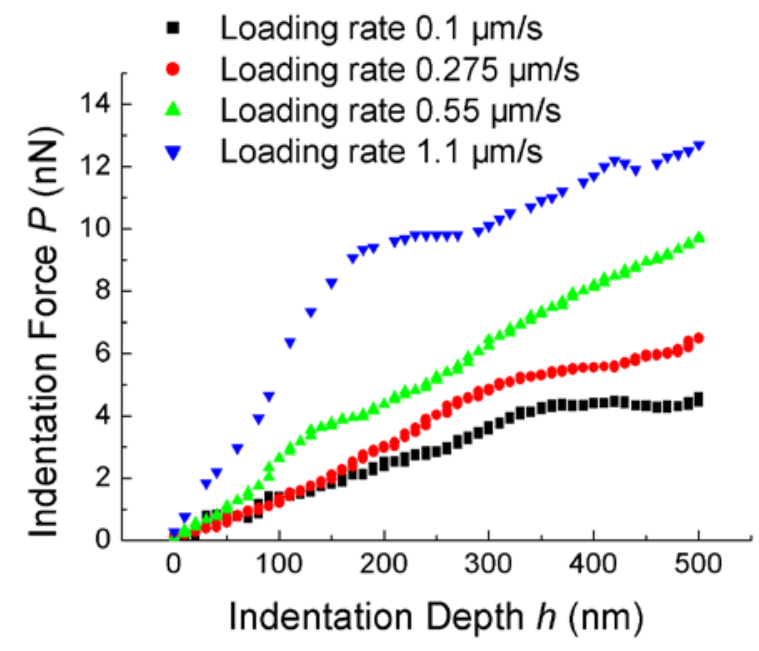

Fig.3a: Indentation force vs indentation depth curve for UM1 cells at different loading rates using the Hertzian indentation protocol. 


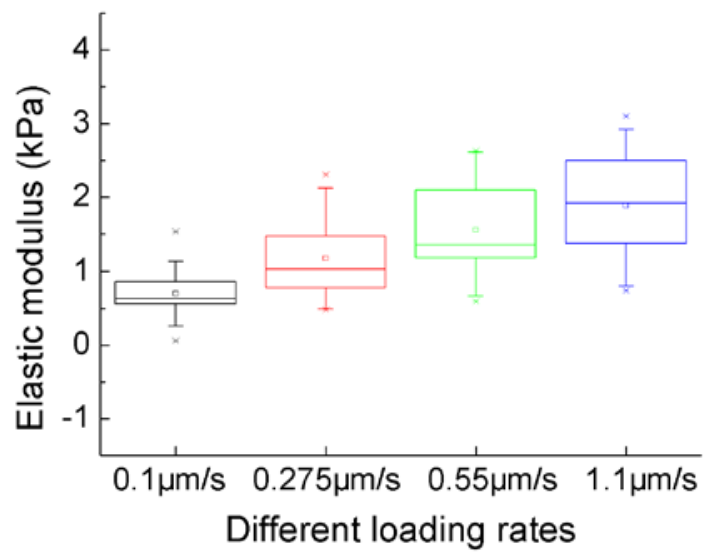

(b)

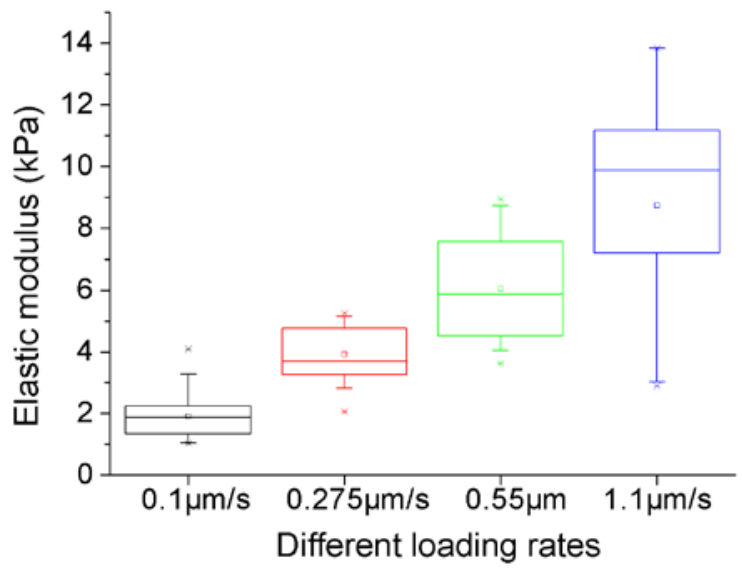

(c)

Fig.3(b,c): Apparent elastic modulus of UM1 cell line at different loading rates calculated with the Hertzian indentation protocol using data up to (b) $100 \mathrm{~nm}$ and (c) $500 \mathrm{~nm}$ of indentation depth. 


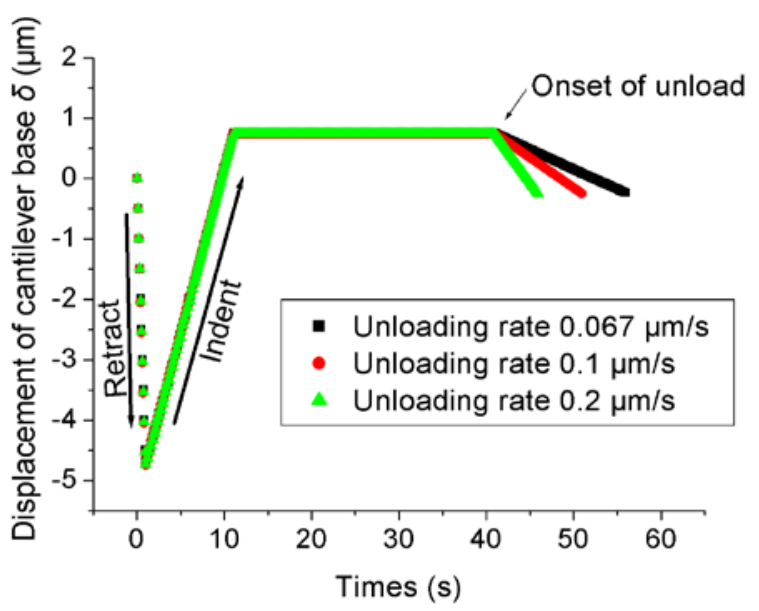

Fig.4a: Displacement of cantilever base vs time curve used in rate jump tests on UM1 cell line. Here, zero height is the set point detected by the machine, $\delta>0$ means downward travel of the cantilever base, and vice versa. The load history is identical up to the end of the hold at $41 \mathrm{~s}$, followed by retraction at rates of $0.2 \mu \mathrm{m} / \mathrm{s}, 0.1 \mu \mathrm{m} / \mathrm{s}$ and $0.067 \mu \mathrm{m} / \mathrm{s}$ respectively.

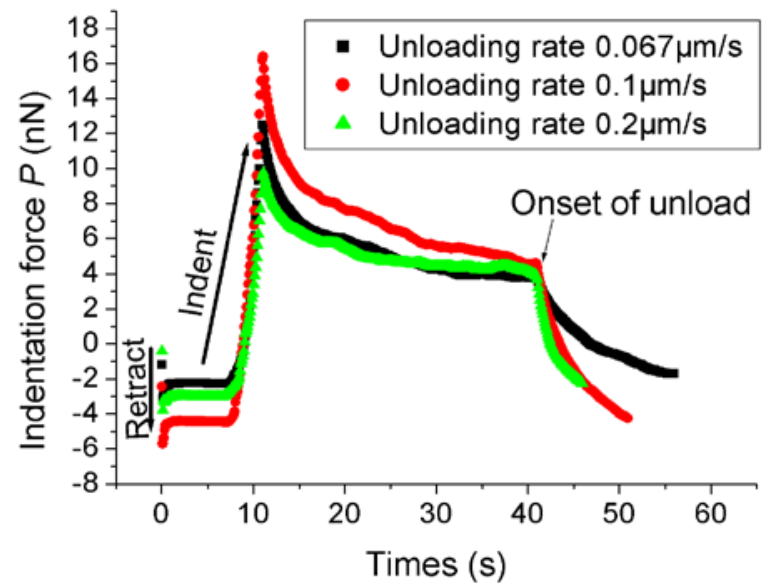

Fig.4b: Resultant indentation force $P$ vs time curve in rate jump tests using the loading profiles in Fig. 4a. 


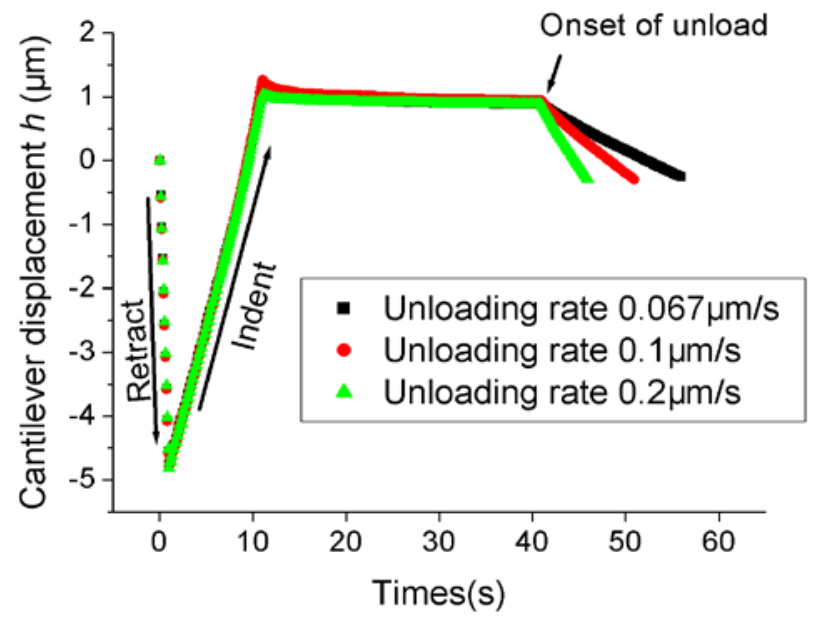

Fig.4c: Resultant displacement $h$ of end of cantilever as detected by the photo-diode vs time in rate jump tests using the loading profiles in Fig. 4a. Zero marks the set point, $h>0$ means downward movement of the tip, and vice versa.

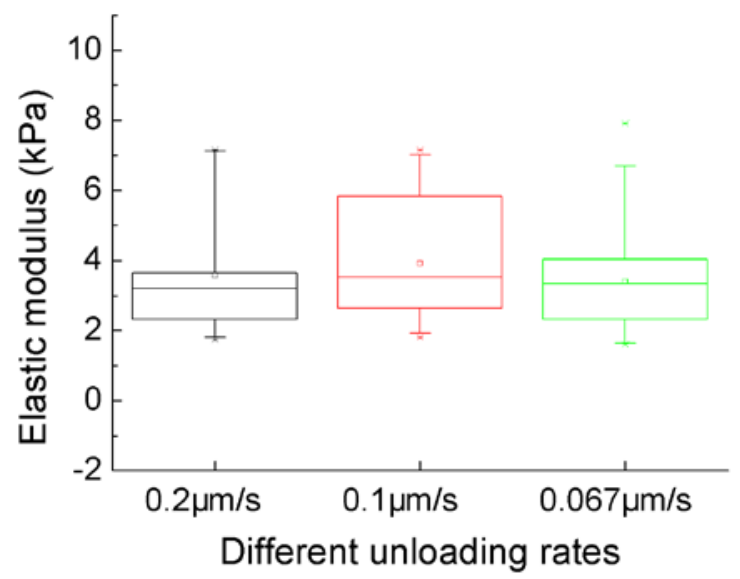

Fig.4d: The elastic modulus of UM1 cell line with different unloading rates in the rate-jump method 